\title{
Metastasios La Clemenza di Tito.
}

Von den am Ende des vorigen Jahrhunderts auch in Deutschland, wie man aus Joh. Adam Hillers 1786 in Leipzig erschienenem Buch Über Metastasio und seine Werke ersehen kann, noch immer hochgerühmten lyrischen Dramen Metastastios hat nur ein einziges, La Clemenza di Tito sich aus der gänzlichen Vergessenheit beim grofsen Publikum gerettet, und das auch nur durch Mozarts Musik. Die von Zeit zu Zeit auf allen guten Bühnen erfolgende Wiederaufnahme der Oper findet stets einen wenn auch nicht dauernden, doch durchschlagenden Erfolg; und wenn man früher wohl die Musik als grofsenteils veraltet angesehen und nur noch etwa zu Festaufführungen für geeignet erklärt hatte, so hat sie doch gerade in der Jetztzeit immer noch eine gröfsere Lebensfähigkeit gezeigt, als man ihr zugetraūt hatte. Deshalb wird eine Auseinandersetzung über die Entstehung des Werkes Metastasios und des darauf gebauten Operntextes sehr angebracht erscheinen, um so mehr als bis heutigen Tages darüber noch nichts veröffentlicht worden, ja, die eigentliche Sachlage bisher noch völlig unbekannt geblieben ist.

Die ganze geschichtliche Grundlage des Metastasioschen Dramas findet sich bei Sueton Titus $9 \mathrm{mit}$ folgenden Worten angegeben: „Zwei junge Patricier, welche überführt worden waren, nach der Kaiserherrschaft gestrebt $\mathrm{zu}$ haben, bestrafte Titus nicht, sondern ermahnte sie nur, von ihrem Vorhaben abzustehen; er versprach sogar, wenn sie sonst Wünsche hegten, sie befriedigen zu wollen; und der besorgten Mutter des einen, welche von Rom abwesend war, liefs er selbst durch einen Eilboten die Nachricht zukommen, dals ihr Sohn begnadigt sei. Übrigens zog er beide junge Männer nicht nur zu seiner Tafel, sondern räumte ihnen auch am folgenden Tage bei einem Gladiatorenspiele Plätze in seiner Nähe ein, ja, er reichte ihnen sogar die ihm vorgelegten Waffen der Kämpfenden zur Ansicht hin. Er soll sodann, als er die Nativität beider kennen gelernt hatte, versichert haben, dafs ihnen eine Gefahr drohe, aber von anderer Seite, wie es denn auch eintrat." Damit bricht Sueton seine Erzählung ab. Dasselbe etwa erzählen Aurelius Victor (de Caes. Io, 3 und Epit. 10, IO), Dio Cassius und Zonaras. Dies ist überhaupt alles, was über den von Metastasio behandelten Gegenstand aus dem Altertum mitgeteilt wird, und der Dichter selbst giebt danach denn auch in dieser knappen Erzählung sein Argomento. Was 
sein lyrisches Drama sonst noch Anderes oder Abweichendes von der Tradition enthält, hat er hineingearbeitet, es ist nur fraglich, $o b$ aus eigner Erfindung oder von einem andern entlehnt.

Es fällt ohne weiteres in die Augen, dafs aus der alten Überlieferung allein, wenn auch ein Konflikt, so doch noch kein Theaterstück sich herstellen liefs. Zudem kann der aus Ehrgeiz unternommene Mord des Herrschers zwar, wie in Macbeth, für eine Tragödie, aber er kann nicht für ein lyrisches Drama zum Vorwurf genommen werden. Deshalb hat Metastasio die Vitellia herbeigezogen, welche, weil ihr Vater von Vespasian entthront worden war und infolge dessen seinen Tod gefunden hatte, aus Rache den Sohn desselben, Titus, zu ermorden oder ermorden zu lassen trachtet und dem Sextus, der sie liebt, nur unter der Bedingung ihre Hand verspricht und Aussicht auf den Thron eröffnet, dafs er ihren blutdürstigen Plan ins Werk setzt. Nun war zwar, nach Sueton Vitellius 6, eine Tochter dieses Kaisers (vielleicht mehrere) vorhanden, aber die Lage derselben war so ganz verschieden von der im Drama des Dichters vorausgesetzten - schon Vespasian hatte sie verheiratet und ausgestattet, Vespasianus 14 - und Titus hatte so wenig Schuld an dem Sturz ihres Vaters, dafs Metastasio bei diesem Sachverhältnis kaum von selbst auf den Einfall geraten konnte, sie die Rolle spielen zu lassen, die er ihr in seinem Stück zuerteilt hat.

Aber freilich hatte er es auch gar nicht nötig, seine Erfindungskraft übermälsig anzustrengen; der ganze Plan des Stücks lag ihm schon anderwärts vor. Er war beauftragt, zur Geburtstagsfeier Karls VI. im Jahre 1734 ein lyrisches Drama zu verfassen, das von Caldara komponiert werden sollte (in der That wurde es mit der Musik dieses Komponisten in jenem Jahre aufgeführt); die Anfangsworte seines Argomento zeigen sofort, dafs er beabsichtigte, die Güte und die Gnade eines Fürsten zum Thema der Festoper zu nehmen; und so richteten sich seine Blicke, um ein Muster dafür zu erhalten, ganz von selbst auf das berühmteste Werk dieser Gattung, auf Corneilles Cinna. Stoff und Verwickelung pafsten ausgezeichnet für seinen Zweck, nur nicht die Person des Augustus; denn abgesehen davon, dals der französische Dichter selbst ihm Grausamkeit und Blutgier vorwerfen lälst, hatte gerade in demselben Jahre, in welchem Metastasio sein Drama schreiben sollte, nämlich 1734, Montesquieu in seinen Considérations sur la grandeur et la décadence des Romains diesen Kaiser als einen kaltherzigen und feigen Tyrannen auf das äufserste in Verruf gebracht. Die beste Persönlichkeit für ein Festspiel dieser Art war unstreitig Titus. Ihn wählte Metastasio und, den oben erwähnten Vorfall zum Anhalt nehmend, versetzte er auf die Zeit dieses Fürsten die übrigen Personen und ihre Verhältnisse aus der Regierung des Augustus, wie sie die Geschichte und das Stück Corneilles vorgezeichnet hatte; aus Cinna ist Sextus, aus Aemilia ist Vitellia gemacht, und die gegenseitige Stellung des ersten Liebespaares auf die beiden letz- 
teren in einigermafsen passender Weise übertragen worden. Wie Aemilia in Cinna, ist Vitellia in La Clemenza di Tito der Haupthebel der Verwickelung, jene allerdings in ihrem Racheschnauben etwas berechtigter, diese weniger, da sie Titus keineswegs die Ermordung ihres Vaters, ja, nicht einmal mit Recht den Verlust seines Thrones zuschreiben kann, weshalb Metastasio noch ihre geheime Absicht, selbst den Kaiser Titus zu heiraten, und ihre Eifersucht auf Berenice herbeizuziehen für nötig gehalten hat. Aus Maximus ist einesteils Lepidus geworden, der ganz im Hintergrund bleibt, andererseits aus ihm Annius entstanden, der nur als Freund des Sextus und als Bewerber um seine Schwester Servilia, deshalb nicht, wie Maximus, als Verschwörungsgenosse und zugleich Nebenbuhler und Mitbewerber um die Hand der Geliebten Cinnas auftritt. Metastasio hat den Anfangsauftritt seines lyrischen Dramas von der vierten Scene des dritten Akts im Stück Corneilles genommen; hier lärst Cinna Aemilia gegenüber seine neuerdings entstandene Unschlüssigkeit, den Plan seiner Verschwörung gegen Augustus auszuführen, durchblicken. Dasselbe thut Sextus in der ersten Scene des ersten Akts bei Metastasio; ihm wirft Vitellia vor:

Onde in te nasce

Questa vicenda eterna

D'ardire e di viltà?

und in seinem der Unterredung zwischen Cinna und Aemilia vorangehenden Monolog sagt der erstere sich:

En ces extrémités quels conseils dois-je prendre?

De quel côté pencher? à quel parti me rendre?

Zum Teil finden sich bei beiden Dichtern dieselben Worte; so sagt Cinna:

Je tremble, je soupire

Mais je n'ose parler;

und Vitellia bemerkt gegen Sextus:

\section{Intender ti vorrei.}

Jener:

Sospiri?

dieser:

J'obéis sans réserve à tous vos sentiments,

non posso

Voler che a voglia tua.

Bei Corneille unterbricht Aemilia Cinna mit den Worten:

C'est trop me gêner, parle;

bei Metastasio heifst es:

Parla, di, che s'attende?

Aus

Ne me condamnez pas

ist

Pria di sgridarmi

Ch'io ti spieghi il mio stato almen concedi. 
Des italienischen Dichters

Vorrei servir ti,

Tradirlo non vorrei

ist nur nicht gerade wörtlich aus dem Monolog Cinnas, der dem vierten Auftritt vorangeht, übersetzt:

Les douceurs de l'amour, celles de la vengeance

N'ont point assez d'appas pour flatter ma raison,

S'il faut les acquérir par une trahison:

Es ist nach Metastasios Annahme durchaus nichts Unwahrscheinliches, dafs Sextus und Vitellia mit den Wohlthaten des Titus, ganz wie Cinna und Aemilia mit den Gunstbeweisen des Augustus, überhäuft worden sind; dals aber jene geradezu den ganzen Genuls der Herrschaft des Titus haben sollen

di sì vasto impero

Tolto l'alloro e l'ostro

Suo tutto il peso e tutto il frutto è nostro,

und dafs Titus selbst erklärt:

Avrai tal parte

Tu ancor nel soglio, e tanto

T'innalzero, che restera ben poco

Dello spazio infinito

Che frapposer gli Dei fra Sesto, e Tito,

das ist denn doch wohl eine aus Corneille II I herübergenommene, bei diesem durch die Vorgänge besser motivierte Übertreibung:

Cinna, par vos conseils je retiendrai l'empire,

Mais je le retiendrai, pour vous en faire part.

Wie die nach vengeance verlangende Aemilia inhumaine und der Kaiser Augustus moins tyran qu'elle, so ist Vitellia, welche chiede yendetta, natürlich barbara, tiranna; während Cinna erklärt:

Mais l'empire inhumain qu'exercent vos beautes.

Force jusqu'aux esprits et jusqu'aux volontés, uft Sextus aus:

Poter della belta I

Oh sovrumano

A poco a poco io perde

L'arbitrio di me stesso.

Vitellia ha in fronte

Un astro che governa il mio destino;

Ind man möchte fast annehmen, dals Metastasio das Buch Correilles vor sich aufgeschlagen gehabt hat, um nicht nur den Inhalt les Auftritts wiederzugeben, nein, um auch seine Ausdrücke daraus sntlehnen zu können. So sagt Aemilia:

Et si pour me gagner il faut trahir ton maître,

Mille autres a l'envi recevraient cette loi,

Ind Vitellia:

A me non manca

Più degno esecutor dell' odio mio; 
und im zweiten Auftritt lehrt uns Annius:

Tito ha l'impero

E del mondo, e di se,

wie Augustus selbst sich rühmt:

Je suis maître de moi, comme de l'univers.

Ja, man kann an diesem Stück besser als an jedem andern desselben Dichters einsehen, dafs Metastasio in seiner ganzen Dichtungsweise, nicht nur im Entwurf und der Komposition seiner Dramen, sondern auch in der Fassung seines Ausdrucks durchaus in der rhetorisch zugeschnittenen Form der klassischen Poesie der Franzosen wurzelt. Hier und da findet man bei ihm Aussprüche, welche an berühmt gewordene Verse eines französischen Dichters erinnern. Das

Le vrai peut quelquefois n'être pas vraisemblable

bringt er in La Clemenza di Tito III 2 mit den Worten an:

quel che vero appare

Sempre vero non è.

Der Schlufs der ersten Scene Metastasios ist natürlich derselbe wie bei Corneille im vierten Auftritt des dritten Akts; hier wird Cinna, dort Sextus überwältigt und überzeugt.

Eh bien, vous le voulez, il faut vous satisfaire,

Il faut affranchir Rome, il faut venger un père

sagt jener, und dieser ebenso:

Tutto, tutto farò. Prescrivi, imponi,

Regola i miei moti.

Zum Überflufs wird auch noch Sextus durch Annius zu Titus beordert, wie es im ersten Akt für Cinna und Maximus durch Evander geschehen war. Alles übrige, was im ersten Akt der Clemenza vorgeht, sowie der ganze zweite Akt gehören, - weil die eigentümlichen von ihm selbst hinzuerfundenen Verhältnisse behandelnd, Metastasio allein an, - wenn man nicht etwa den Rat, den Vitellia dem Sextus giebt, zu fliehen, aus dem Vorschlage, den Maximus der Aemilia macht, mit ihm zusammen zu entweichen, entlehnt glauben will.

Der dritte Akt der Clemenza di Tito lenkt dagegen wieder in das Fahrwasser des Cinna ein, aber während Corneille die Entwickelung von der stattlichen Fregatte seines fünften Aktes tragen lälst, hat Metastasio sie auf eine ganze Anzahl kleiner Barken zersplittert, ich will sagen, sie auf eine ganze Reihe nicht einmal örtlich zusammenhängender und durch Monologe mehrfach unterbrochener Scenen verteilt. Der letzte Akt Cinnas behält, trotz der veralteten Form, immer noch etwas Majestätisches; im Titus, mögen immerhin die Beweggründe seines Helden edler sein als bei Augustus, erhält die Verzeihung doch etwas gar zu Weichliches, Sentimentales und Weibisches. Auch in Metastasios Stück klagt sich endlich Vitellia - aber freilich ihrem unentschiedenen Wollen und schwan- 
kenden Charakter gemäls, erst als sie sich entdeckt glaubt, - wie Aemilia bei Corneille an. Bei der gleichen Entwickelung kehren auch die ähnlichen Ausdrücke zurück. Wenn Titus ausruft:

Che orror! che tradimento!

Che nera infedelta! Fingersi amico,

Essermi sempre al fianco! ogni momento

Esiger dal mio core

Qualche prova d'amore; e starmi intanto

Preparando la morte! Ed io sospendo

Ancor la pena. E la sentenza ancora

Non segno. Ah si, lo scellerato mora!

so ist das doch nur eine Umschreibung der Worte Corneilles IV r:

Quoi! mes plus chers amis! quoi, Cinna! quoi, Maxime!

Les deux que j'honorais d'une si haute estime,

À qui j'ouvrais mon cœur, et dont $j$ 'avais fait choix

Pour les plus importants et plus nobles emplois!

Après qu'entre leurs mains j'ai remis mon empire,

Pour m'arracher le jour l'un et l'autre conspire

(IV 3) Punissons l'assassin, proscrivons les complices.

In $V_{2}$ fängt Aemilia ihre Selbstanklage an:

Oui, tout ce qu'il a fait, il l'a fait pour me plaire;

Et j'en étais, seigneur, la cause et le salaire.

Je ne voulus jamais lui donner d'espérance,

Qu'il ne m'eat de mon père assuré la vengeance;

Vitellia dagegen $\mathrm{III}_{1} 3$ :

Io la più rea

Son di ciascuno; io meditai la trama, 11 più fedele amico

Io ti sedussi; io del suo cieco amore

A tuo danno abusai.

Während Augustus verwundert und unwillig fragt:

En est-ce assez, ô ciel! et le sort pour me nuire

A-t-il quelqu'un des miens qu'il veuille encor séduire? etc.

fragt Titus III 13 :

Ma che giorno è mai questo? Al punto istesso

Che assolvo un reo, ne scopro un altro etc.

Bei Corneille ruft Augustus die Nachwelt an:

$\mathrm{O}$ siècles, $\hat{\mathbf{~ m e ́ m o i r e}}$

Conservez à jamais ma dernière victoire, 
und Titus überlegt:

$$
\begin{aligned}
& \text { Or che diranno } \\
& \text { I posteri di noi. }
\end{aligned}
$$

Aemilia und Vitellia versichern beide, die eine:

Ma haine va mourir que j'ai crue immortelle,

L'ardeur de vous servir succède à sa fureur,

die andere:

$$
\text { - In fin ch' io viva }
$$

Fia sempre il tuo voler legge al mio core.

Gleiches thun ferner Cinna und Sextus:

Seigneur, que vous dirai-je, après que nos offenses

Au lieu de châtiments trouvent des récompenses?

$O$ vertu sans exemple, ô clémence qui rend

Votre pouvoir plus juste, et mon crime plus grand.

Ah Césare! ah Signore! $\mathrm{E}$ poi non soffri

Ch' adori la terra ....

E come, e quando

Sperar potrò che la memoria amara

De' falli miei ....

und Titus antwortet:

wie Augustus:

$$
\text { Sesto, non più, }
$$

Cesse d'en retarder un oubli magnanimé:

und wie Augustus Cinna auffordert:

Soyons amis, Cinna,

so Titus den Sextus:

$$
\text { torniamo }
$$

Di nuovo amici.

Wer die Clemenza di Tito nur aus dem Mozarts Oper zu Grunde liegenden Libretto kennt, dem kann es natürlich nicht aufgefallen sein, wie sehr das Werk Metastasios sich an die französische Tragödie anlehnt; denn die bezeichnendsten Stellen sind im Operntext eben weggeblieben und die Anordnung des Stücks überhaupt ist sehr verändert. "Mazzola (der Verfasser des Textbuches) hat", so führt Jahn, Mozarts Leben und Werke IV 572, an, „nur die Nummern 2, 5, 6, 8, 9, 1 1, 16, 20, 21,25 , sowie die Recitative I I, 1 7, 22, 24 aus Metastasio herübergenommen; er hat die Arien des Annius 13, 17, des Sextus 19, der Vitellia 23, die Duette 1, 3, 7, die Terzette 10, 14, 18, das Quintett 12, das Sextett 26 und den Chor 15 hinzugedichtet; meistens mit teilweiser Benutzung von Metastasios Motiven, auch wohl mit Beibehaltung einzelner Verse und Wendungen."

Was Jahn hiermit summarisch beibringt, werde ich wenigstens an einem Beispiel belegen. 
Mit Unterdrückung von beinahe vier Seiten in Metastasios erster Scene fängt Mazzola die Oper mit den Worten an, denen in jenes lyrischem Drama die gegenüberstehenden Zeilen entsprechen:

Sesto.

Come ti piace, imponi,

Regola i moti miei.

Il mio destin tu sei;

Tutto farò per te.

Vitellia.

Prima che il sol tramonti, Estinto io vo' l' indegno.

Sai ch' egli usurpa un regno

$\mathrm{Ch}$ ' in sorte il ciel mi diè.

\section{Sesto.}

Gia il tuo furor m'accende.

Vitellia.

Ebben, che più s'attende.

Sesto.

Un dolce sguardo almeno

Sia premio alla mia fe.

Vitellia. Sesto.

Fan mille affetti insieme

Battaglia in me spietata;

Un' alma lacerata

Più della mia non v'è.
Sesto.

Tutto, tutto farò. Precrivi, imponi, Regola i miei moti.

Tu ha mia sorte, il mio destin tu sei.

\section{Vitellia.}

Prima che il sol tramonti

Voglio Tito svenato etc.

(vorher) e più non pensi

Che questo Eroe clemente un soglio usurpa

Dal suo tolto al mio padre.

Sesto.
(vorher) rapir mi sento
Tutto nel tuo furor.

Vitellia.

Parla, di, che s'attende.

Die deutsche Bearbeitung des Textes stimmt nicht überall, was wenig $\mathrm{zu}$ bedauern sein würde, mit den italienischen Worten überein, aber, was schlimmer ist, sie palst oft nicht zu der Musik, die nach ihnen gesetzt ist. Am auffallendsten erscheint das in No. 9. Die Worte

\footnotetext{
Parto, ma tu, ben mio,

Meco ritorna in pace
}

sind elegisch angehaucht, wie es das eben wieder erfolgte Nachgeben des Sextus und sein Wunsch, trotz inneren Widerstrebens, Vitellia zufrieden zu stellen, erfordert, und das drückt die Musik aus: sie malt in den Anfangsnoten den erzwungenen heroischen Aufschwung des Sextus. Aber dazu ist die deutsche Übersetzung

Feurig eil' ich zur Rache! 
ganz ungeeignet gewählt. Die Pause namentlich zwischen den Worte "feurig“ und der Wiederholung desselben ist unnatürlich und wer Mozart nach dem Ausdruck, welchen seine Noten del Worten geben, beurteilt, muls an dem Feuer, welches er in dit Musik zu legen im stande war, höchlich zweifeln; auch die Prä. position "zur" auf der höchsten Note der musikalischen Stropht kann leicht zu einem von den Fällen gerechnet werden, wo mal dem Komponisten eine unrichtige Betonung vorwerfen zu könnel glaubt. Eine andere deutsche Bearbeitung, welche stellenweise der Text bessert, würde bei uns der Oper zum Vorteil gereichen.

H. J. HeLler. 